\title{
Self-rated health, quality of life and appetite as predictors of initiation of dialysis and mortality in patients with chronic kidney disease stages 4-5: a prospective cohort study
}

Birgith Engelst Grove ${ }^{1 *} \mathbb{0}$, Liv Marit Schougaard², Niels Henrik Hjollund ${ }^{3,4}$ and Per Ivarsen ${ }^{5}$

\begin{abstract}
Objective: Patient-reported health status, including symptom burden, functional status and quality of life, are important measures of health in patients with chronic kidney disease. We aimed to investigate patient-reported outcomes (PRO) on self-rated health, appetite, quality of life and their associations with clinical outcomes. We conducted a prospective observational cohort study. Data was collected at baseline using a PRO questionnaire. The primary outcomes were initiation of dialysis, transplantation and mortality. Kaplan-Meier curves and multivariable Cox proportional hazards regression analyses were used.

Results: A total of 126 patients with chronic kidney disease with an eGFR of $\leq 25 \mathrm{~mL} / \mathrm{min} / 1.73 \mathrm{~m}^{2}$ were followed for a median of 321 (range 10-523) days. Poor appetite was associated with mortality (hazard ratio 20.9, 95\% Cl 3.7129.8). Initiation of dialysis was associated with low self-rated health (hazard ratio 5.2, 95\% Cl 1.2-24.0). Mean decline in estimated glomerular filtration rate was $-0.9 \mathrm{~mL} / \mathrm{min} / 1.73 \mathrm{~m}^{2}(95 \% \mathrm{Cl}-1.6$ to -0.2$)$. Decline in self-rated health $(p=0.001)$ and appetite $(p=0.002)$ were correlated with reduction in renal function.
\end{abstract}

Keywords: Patient-reported outcome, Chronic kidney disease, Self-reported health, Quality of life, Pre-dialysis, Initiating dialysis, Transplantation, Mortality

\section{Introduction}

Chronic kidney disease (CKD) is increasing globally as a consequence of people getting older, the increased prevalence of diabetes, hypertension and obesity and affects approximately $10 \%$ of the adult population [1]. Patients suffering from CKD need to modify their diet and lifestyle and attend regular medical appointments. A method to gain knowledge directly from the patients is through patient-reported outcome measures (PRO's), which is defined as a measurement based on "any report

\footnotetext{
*Correspondence: bigcri@rm.dk

${ }^{1}$ Department of Renal Medicine, Aarhus University Hospital \&

AmbuFlex/WestChronic, Regional Hospital West Jutland, Gl.Landevej 61,

7400 Herning, Denmark

Full list of author information is available at the end of the article
}

of the status of a patient's health condition that comes directly from the patient, without interpretation of the patient's response by a clinician or anyone else" [2]. Use of PROs has increased in clinical practice with the recognition that measuring health as, for example, mortality and time to dialysis initiation, does not cover the complete perspective of the patient perception of health [3]. A recent comprehensive review concluded that use of PROs improves patient-centred care and supports clinical decisions [4]. Previous cohort studies in dialysis patients have suggested an association between self-reported health, quality of life (QoL), morbidity and mortality [5-7]. However, few studies have included patients prior to the onset of dialysis, and to the best of our knowledge none has investigated the relationship between PRO measures 
and renal function. The aim of this study was to investigate the association between PROs (self-rated health, appetite, quality of life) on initiation of dialysis, transplantation and mortality in patients with chronic kidney disease. We hypothesised that pre-dialysis patients with a low score in self-rated health or low QoL or poor appetite had an increased risk of death and initiation of dialysis during the follow-up period. Secondly, we aimed to describe the longitudinal changes in self-reported health and to compare these with changes in eGFR in a subgroup of patients' not starting renal replacement therapy during follow-up.

\section{Main text \\ Method \\ Study population}

In total, 126 prevalent patients were included, in this prospective cohort study, from the outpatient clinic at Department of Renal Medicine, Aarhus University Hospital, Denmark from October 2013 to March 2014. Patients were eligible for inclusion if they were more than 18 years of age, had an eGFR of $25 \mathrm{~mL} / \mathrm{min} / 1.73 \mathrm{~m}^{2}$ or less and were candidates for active uraemia treatment. Exclusion criteria were cognitive impairment, visually impairment, incapable of using a touch screen or computer or having language difficulties. Patients were recruited in connection with their regular visits at the outpatient clinic, and sample size was based on feasibility rather than formal power calculations. A retrospective analysis revealed that the clinic in this inclusion period comprised 703 patients with an eGFR $\leq 25 \mathrm{~mL} / \mathrm{min}$. No significant differences were found between participants and non-participants with respect to age, gender and renal function (data not shown).

\section{Data collection}

The patients completed a questionnaire either at home or on a touch screen available at the clinic prior to each consultation; this was a part of daily routine clinical practice. The questionnaire consisted of a single item from SF-36 about self-rated health [8] and EQ-5D [9], which includes five multi-attribute items on QoL together with a kidney failure related question on self-rated appetite from the Kidney Disease and Quality of Life ${ }^{\mathrm{TM}}$ Short Form questionnaire [10]. The questionnaire was developed for clinical practice in collaboration between patients in the nephrology clinic and a group of experts consisting of physicians, a dietician, nurses working in the nephrology clinic and AmbuFlex. See Additional file 1: The Questionnaire. AmbuFlex is a web-administered PRO system and a method to use PRO in clinical practice where regularly scheduled follow-ups are substituted or supplemented with regular diagnosis-specific electronic questionnaires filled in by the patient prior consultation [11]. Staff nurses and the author (BEG) included patients consecutively. A graphical overview of the patients' answers was available for the clinicians in the Electronic Medical Record (Additional file 2: Figure S1).

The endpoints were grouped into the four mutually exclusive categories: still in pre-dialysis care, in dialysis, transplanted and deceased (Table 2). Analyses of change in PRO measures in patients still in pre-dialysis at the end of follow-up included 42 patients. Time between filling in of the questionnaires was median 375 (IQR 322;488) days. 34 patients were lost to follow-up due to incomplete filling in of the questionnaire.

\section{Variables}

Clinical and demographic data were collected from medical records. Primary cause of kidney disease was classified using the ICD-10 codes (International Classification of Diseases) in accordance with previous national calculations [12]. Comorbidity was determined from chart reviews and categorised according to the Charlson Comorbidity Index (CCI) [13]. Laboratory measurements comprising estimated GFR calculated according to the MDRD 4-point formula [14]. Creatinine, albumin and haemoglobin were obtained from the Department of Clinical Biochemistry, Aarhus University Hospital. Blood samples were taken as part of routine practice at baseline and at follow-up, with an average time span from blood sampling to completion of the questionnaire of median 3 (range 1-7) days.

\section{Statistical analyses}

PRO data on appetite "How would you rate your appetite?" and health "In general, would you say your health is?" were categorised from five possible responses into three categories (excellent/very good, good, fair/poor). This was done because of the low incidences in the most extreme categories, as has been done in previous studies [5, 15]. Quality of life (QoL) was measured using the continuous EQ-5D score, which was categorised at the 25th and 75th percentile into three categories 'very good', 'good' and 'low' QoL in concordance with statistical recommendations. Baseline characteristics were presented for the total study population. Normally distributed variables were presented as mean \pm standard deviation (SD), skewed distributed variables were presented as median with interquartile range (IQR). Kruskal-Wallis, $t$ tests and $\mathrm{X}^{2}$ tests were used when appropriate. The analysis for the longitudinal change in PRO data was performed using the Wilcoxon signed-matched pair test. Kaplan-Meier curves were used to illustrate the proportion of participants reaching one of the endpoints during follow-up [16]. 
A Cox model adjusted for baseline covariates was estimated to determine the association between baseline PRO factors and dialysis, transplantation or mortality, with 'excellent/very good' as the reference category. $p$ values of less than 0.05 were considered statistically significant. All analyses were performed in STATA statistical software, version 11.2.

\section{Results}

\section{Patient characteristics}

Characteristics of the total study population are outlined in Table 1 . At baseline, $35 \%$ of the study population rated their health as poor, 25\% reported low QoL and 6\% reported poor appetite. The majority of patients $(60.3 \%)$ were still in pre-dialysis care, while the rest of the patents were almost equally distributed among the three other endpoint categories (Table 2).

Table 1 Baseline characteristics of study sample $(\mathrm{N}=126)$ (Results/patient characteristics)

\begin{tabular}{|c|c|c|}
\hline Characteristics & Total & \\
\hline Age in years, median & 68.5 & {$[22]$} \\
\hline Men, n (\%) & 83 & $(66)$ \\
\hline Weight (kg) & 76 & [17] \\
\hline $\mathrm{BMI}\left(\mathrm{kg} / \mathrm{m}^{2}\right)$ & 26 & {$[6.5$} \\
\hline Systolic BP (mmHg) & 138 & {$[17]$} \\
\hline Diastolic BP (mmHg) & 78 & {$[11]$} \\
\hline eGFR (mL/min), median & 14.5 & {$[8]$} \\
\hline Primary kidney disease $^{a}$ & $\mathrm{~N}$ & $\%$ \\
\hline Diabetes & 23 & (18) \\
\hline Polycystic KD & 11 & (9) \\
\hline Hypertension & 9 & (7) \\
\hline Kidney transplanted & 9 & $(7)$ \\
\hline Glomerulonephritis & 5 & $(4)$ \\
\hline Vasculitis & 4 & (3) \\
\hline Chronic interstitial nephritis & 3 & $(2)$ \\
\hline Other $^{b}$ & 9 & (7) \\
\hline Unknown & 53 & $(42)$ \\
\hline \multicolumn{3}{|l|}{ Medication intake } \\
\hline$>9$ drugs per day & 54 & $(43)$ \\
\hline$\leq 9$ drugs per day & 72 & $(57)$ \\
\hline Use of phosphate binder & 37 & (29) \\
\hline Use of erythropoietin & 56 & $(44)$ \\
\hline \multicolumn{3}{|l|}{ Charlson Comorbidity Index (CCI) } \\
\hline Low (0-2) & 50 & $(40)$ \\
\hline Medium (3-5) & 63 & (50) \\
\hline $\operatorname{High}(\geq 6)$ & 13 & (10) \\
\hline
\end{tabular}

\section{Mortality}

In total $13(10.3 \%)$ of the patients died during followup. Compared to patients who remained in pre-dialysis, those who died during follow-up were more likely to rate their health as fair/poor, their appetite as fair/poor and their QoL as low (Table 2). Time until death is outlined in Kaplan-Meier curves, see Additional file 3: Figure S2. After adjustment for age, sex and comorbidity, only poor appetite remained significantly associated with increased risk of mortality (Table 3).

\section{Patients initiating dialysis}

Twenty-one (16.7\%) patients began dialysis during follow-up. Those who initiated dialysis during follow-up had a lower baseline eGFR compared to patients still in pre-dialysis care $(p<0.001)$ and a higher burden of comorbidity $(p=0.02)$ (Table 2$)$. Patients with poor appetite, low QoL and poor self-rated health tended to initiate dialyses earlier, but no significant difference was found (Additional file 3: Figure S2). Patients reporting fair/poor health at baseline had an increased risk of initiating dialysis during follow-up (HR 5.18, 95\% CI 1.12-23.95). Selfreported appetite or QoL did not associate with initiating dialysis (Table 3).

\section{Transplantation}

In total, 16 (13\%) patients had a kidney transplant during follow-up. We found a shorter time to transplantation among the patients reporting poor health but no difference in appetite and QoL (Additional file 3: Figure S2). In the adjusted analysis we found no significant associations between self-rated health, appetite, QoL and transplantation (Table 3).

\section{Change in renal function and the association with change in PRO data}

A significant decline in eGFR $(p=0.02)$ from baseline until end of follow-up was found in patients $(n=76)$ still in pre-dialysis care. Mean decline in eGFR was $-0.89 \mathrm{~mL} / \mathrm{min} / 1.73 \mathrm{~m}^{2}$ (95\% CI -1.59 to -0.19$)$. A decline in eGFR was seen in 41 patients, 11 patients had an unchanged eGFR during follow-up, and eGFR increased in 24 patients. No significant differences were seen in serum albumin and haemoglobin (Additional file 4: Table S1). Patients with a decline in eGFR $(n=27)$ during follow-up reported an increased loss of appetite $(p=0.002)$ and decreased self-rated health $(p=0.001)$. QoL did not change. Among patients with a steady or an increased eGFR $(n=15)$, no significant difference in selfrated appetite, health or QoL was found. Adjustment for age, sex and comorbidity did not change the estimates (data not shown). 
Table 2 Baseline characteristics in 126 patients stratified by reached endpoint after follow-up

\begin{tabular}{|c|c|c|c|c|c|c|c|}
\hline \multirow[b]{2}{*}{ Characteristics } & \multirow{2}{*}{$\begin{array}{l}\text { Pre-dialyses patients } \\
\mathrm{N}=76^{\mathrm{a}} \\
\text { Median [IQR] }\end{array}$} & \multicolumn{2}{|c|}{$\begin{array}{l}\text { Dialyses patients } \\
N=21\end{array}$} & \multicolumn{2}{|c|}{$\begin{array}{l}\text { Kidney transplanted } \\
\mathrm{N}=16\end{array}$} & \multicolumn{2}{|c|}{$\begin{array}{l}\text { Deceased patients } \\
N=13\end{array}$} \\
\hline & & Median [IQR] & p value ${ }^{b}$ & Median [IQR] & p value ${ }^{c}$ & Median [IQR] & p value ${ }^{d}$ \\
\hline eGFR $\left(\mathrm{mL} / \mathrm{min} / 1.73 \mathrm{~m}^{2}\right)$ & $17[8]$ & $12[4]$ & $<0.001$ & $11.5[3]$ & $<0.001$ & $16[6]$ & 0.38 \\
\hline Serum albumin (g/L) & $37[6]^{5}$ & $34[4]$ & 0.03 & 38 [3] & 0.9 & $36[11]$ & 0.31 \\
\hline $\mathrm{CCl}$ & $3[2]$ & $4[2]$ & 0.02 & $2[1]$ & 0.73 & $4[2]$ & 0.06 \\
\hline Men, n (\%) & $44(83)$ & $16(76)$ & 0.13 & $14(88)$ & 0.03 & $9(69)$ & 0.44 \\
\hline Age, years & $72[17]$ & $62[15]$ & 0.14 & $47[16]$ & $<0.001$ & $81[10]$ & 0.31 \\
\hline Drugs pEr day & $8[69]$ & $10[5]$ & 0.02 & $8[5]$ & 0.40 & $10[8]$ & 0.28 \\
\hline Erythropoietin, n (\%) & $26(34)$ & $14(67)$ & $<0.01$ & $8(50)$ & 0.23 & $8(24)$ & 0.06 \\
\hline Phosphate binders, n (\%) & $17(22)$ & $10(48)$ & 0.03 & $6(37)$ & 0.21 & $4(20)$ & 0.51 \\
\hline Self-rated health & $\mathrm{N}(\%)$ & N (\%) & & $\mathrm{N}(\%)$ & & N (\%) & \\
\hline Excellent/very good & $19(25)$ & $2(10)$ & & $4(25)$ & & $1(8)$ & \\
\hline Good & $38(50)$ & $7(33)$ & & $7(44)$ & & $4(31)$ & \\
\hline Fair/poor & $19(25)$ & $12(57)$ & 0.02 & $5(31)$ & 0.65 & $8(62)$ & 0.03 \\
\hline \multicolumn{8}{|l|}{ Self-rated appetite } \\
\hline Excellent/very good & $54(71)$ & $9(43)$ & & $9(56)$ & & $6(46)$ & \\
\hline Good & $19(25)$ & $11(52)$ & & $7(44)$ & & $4(31)$ & \\
\hline Fair/poor & $3(4)$ & $1(5)$ & 0.05 & - & 0.78 & $3(23)$ & 0.03 \\
\hline \multicolumn{8}{|l|}{ Quality of life } \\
\hline Very good & $18(24)$ & $6(29)$ & & $6(38)$ & & - & \\
\hline Good & $42(55)$ & $8(38)$ & & $9(56)$ & & $5(38)$ & \\
\hline Low & $16(21)$ & $7(33)$ & 0.34 & $1(6)$ & 0.05 & $8(62)$ & 0.01 \\
\hline
\end{tabular}

CCl Charlson Comorbidity Index

a Patients still in pre-dialysis care

b Difference between dialysis starters and pre-dialysis group

c Difference between the transplanted and pre-dialysis group

d Difference between deceased and pre-dialysis group

e 1 missing

Table 3 Adjusted hazard ratio for mortality, initiating dialysis and transplantation associated with self-rated health, appetite and quality of life. $\mathrm{N}=126$

\begin{tabular}{|c|c|c|c|}
\hline PRO & Health & Appetite & QoL \\
\hline \multicolumn{4}{|c|}{ Adjusted Hazard Ratio for mortality $(n=13)$} \\
\hline Excellent/very good & 1 (reference) & 1 (reference) & 1 (reference) \\
\hline Good & $0.98(0.10-9.24)$ & $0.91(0.20-4.08)$ & $0.35(0.09-1.39)$ \\
\hline Fair/poor & $3.41(0.41-28.1)$ & $20.78^{*}(3.46-124.71)$ & $2.88(0.71-11.58)$ \\
\hline \multicolumn{4}{|c|}{ Adjusted Hazard Ratio for initiation of dialysis $(n=21)$} \\
\hline Excellent/very good & 1 (reference) & 1 (reference) & 1 (reference) \\
\hline Good & $2.25(0.46 ; 11.1)$ & $2.47(0.99 ; 6.23)$ & $0.66(0.23 ; 1.98)$ \\
\hline Fair/poor & $5.18^{*}(1.12 ; 23.95)$ & $1.59(0.19 ; 12.98)$ & $1.22(0.37 ; 3.93)$ \\
\hline \multicolumn{4}{|c|}{ Adjusted Hazard Ratio for receiving a kidney transplant $(n=16)$} \\
\hline Excellent/very good & 1 (reference) & 1 (reference) & 1 (reference) \\
\hline Good & $1.68(0.47 ; 6.06)$ & $1.37(0.50 ; 3.80)$ & $1.16(0.40 ; 3.42)$ \\
\hline Fair/poor & $1.37(0.34 ; 5.51)$ & - & $0.32(0.03 ; 3.43)$ \\
\hline
\end{tabular}

Numbers in table are Hazard Ratio (95\% confidence interval). QoL quality of life

Adjusted for age, sex and comorbidity. ${ }^{*} \mathrm{p}<0.05$ 


\section{Discussion \\ Main findings}

In this prospective cohort study, poor appetite predicted increased risk of mortality and poor self-rated health was a predictor of initiation of dialyses in patients with CKD stage 4-5. We did not find any associations between PRO and transplantation. Decline in renal function was associated with decreased self-rated health and appetite.

\section{Mortality}

The mortality rate among pre-dialysis patients, was similar to previous findings reported in other pre-dialysis and dialysis populations $[5,17]$. QoL was not associated with mortality. Previous studies have shown that QoL is a good indicator of physical well-being in a healthy elderly population as well as in patients on peritoneal dialysis, and QoL appears to be a strong predictor of morbidity and mortality [7, 18]. These studies used the SF-36 measurement tool and not the EQ-5D as done in present study. Recent data from the CRIC cohort including 3837 patients with CKD stages 2-4, with a follow-up time of 6.2 years demonstrated that low HRQoL, using the SF-36 measurement tool, was associated with increased risk of incident cardiovascular events and death but not CKD progression [19]. Interestingly, we found no significant association between self-rated health and mortality in present study. This may reflect our relatively small data set or the difference in follow-up time. In the adjusted analysis, in the present study, self-rated appetite was the only significant predictor of mortality, but confidence intervals were wide, probably due to a low number of events. In concordance with other studies, we also demonstrated that patients with poor appetite had low p-albumin, which in several previous studies has been shown to be a strong marker of mortality [15, 20, 21].

\section{Initiating dialysis}

Patients initiating dialysis reported a lower self-rated health compared to patients still in pre-dialysis care, which might reflect the burden of their symptoms related to the progression of renal failure. This finding concurs with a Japanese study among 471 patients with kidney failure, which showed a positive relation between loss of renal function and decline in several domains of SF-36 [22]. We found no association between QoL and initiation of dialysis. A similar study in a UK pre-dialysis population demonstrated a higher risk for death but not progression to End-Stage Renal Disease among those with a lower EQ-5D score [23].

\section{Transplantation}

We found no significant associations PRO measures and transplantation. The decision of transplantation relies mostly on availability of a suitable donor and therefore we did not have an a priori hypothesis concerning this endpoint. The negative result was therefore expected.

\section{Change in renal function and the association with change in PRO data}

Patients who had a decline in eGFR also experienced a decreased appetite and lower self-rated health. This indicates a relationship between eGFR and these PROs, which might be useful information to the clinicians when decision of future treatment is made together with the patient.

We found an association between self-reported low appetite and mortality during follow-up. Furthermore, low self-reported health was a predictor of initiating dialysis. In the context of the study limitations, our findings may indicate that implementing PRO in clinical practice could help identifying symptoms burden and prevent health impairment. Despite study limitations, the results of this study represent a step forward in exploring the use of PRO in a renal failure population. A randomised trial to investigate the implications of the use of PROs in this population has now been designed with a planned initiation of study enrolment September 2018.

\section{Limitations}

This study had limitations such as a rather small sample size, as reflected by the wide confidence intervals. This limited our opportunity for statistical comparisons across disease stage and adjustment could only be performed for the most basic variables, thus residual confounding cannot be ruled out. This reduced our statistical power and accuracy of the results. The recruitment method and the relatively large number of non-participants may have introduced selection bias and thus reduced the external validity of our findings, however our drop out analysis showed no differences between participants and nonparticipants. The study was based only on a single institution, thus limiting the generalisability to other settings or different populations such as dialysis- or transplant patients. Accordingly, caution should be taken when interpreting our findings.

\section{Additional files}

Additional file 1. The Questionnaire (Method/data collection).

Additional file 2: Figure S1. Graphical overview of patient reported outcome in the electronic medical journal (Method/data collection).

Additional file 3: Figure S2. Kaplan-Meier plot for initiation of dialysis, kidney transplantation and mortality (Results).

Additional file 4: Table S1. Laboratory values at baseline and after follow-up ( $\mathrm{N}=76$ ) (Results/Change in renal function and the association with change in PRO data). 


\section{Abbreviations}

eGFR: estimated glomerular filtrations rate; QoL: quality of life; CCl: Charlson Comorbidity Index; ESRD: End Stage Renal Disease; CKD: chronic kidney disease; HRQoL: health related quality of life.

\section{Authors' contributions}

BEG conceived and designed the study, performed the statistical analysis, analysed the results and drafted the manuscript. PI participated in designing the study, drafting the manuscript and analysing the results. LMS and $\mathrm{NHH}$ participated in drafting the manuscript and revised it critically for important intellectual content and contributed to the acquisition of data. All authors read and approved the final manuscript.

\section{Authors' information}

The first author BEG, is an employee at Ambuflex and has previously been employed at Department of Renal Medicine, both of which are parts of the public hospital.

\section{Author details}

1 Department of Renal Medicine, Aarhus University Hospital \& AmbuFlex/ WestChronic, Regional Hospital West Jutland, Gl.Landevej 61, 7400 Herning, Denmark. ${ }^{2}$ AmbuFlex/WestChronic, Regional Hospital West Jutland, Gl. Landevej 61, 7400 Herning, Denmark. ${ }^{3}$ AmbuFlex/WestChronic, Occupational Medicine, University Research Clinic, Health, Aarhus University, Gl. Landevej 61, 7400 Herning, Denmark. ${ }^{4}$ Department of Clinical Epidemiology, Aarhus University Hospital, Aarhus, Denmark. ${ }^{5}$ Department of Renal Medicine, Aarhus University Hospital, Palle Juul-Jensens Boulevard 99, 8200 Aarhus N, Denmark.

\section{Acknowledgements}

We thank all study participants for providing data to this study. The authors thank the Department of Renal Medicine at Aarhus University Hospital in the Central Denmark Region for practical support.

\section{Competing interests}

The authors declare that they have no competing interests.

\section{Availability of data and materials}

The datasets used during the current study are available from the corresponding author on request.

\section{Consent for publication}

Not applicable.

\section{Ethics approval and consent to participate}

The Danish Data Protection Agency (Jr 1-16-02-619-14) and the Danish Health and Medicines Authority (Jr 3-3013-760/1/) have approved the study without the need for obtaining the informed consent.

\section{Funding}

The Kidney Association, Denmark, kindly supported this publication.

\section{Publisher's Note}

Springer Nature remains neutral with regard to jurisdictional claims in published maps and institutional affiliations.

Received: 10 November 2017 Accepted: 4 June 2018

Published online: 08 June 2018

\section{References}

1. Collins AJ, Foley RN, Chavers B, Gilbertson D, Herzog C, Ishani A, et al. US Renal Data System 2013 annual data report. Am J Kidney Dis. 2014;63(1 Suppl):A7.

2. Food and Drug Administration. Food and Drug Administration patientreported outcome measures: use in medicinal product development to support labelling claims. 2013; http://www.fda.gov/downloads/Drugs/ GuidanceComplianceRegulatoryInformation/Guidances/UCM193282.pdf. Accessed 24 Oct 2016.

3. Valderas JM, Kotzeva A, Espallargues M, Guyatt G, Ferrans CE, Halyard MY, et al. The impact of measuring patient-reported outcomes in clinical practice: a systematic review of the literature. Qual Life Res. 2008;17(2):179-93.

4. Johansen MA, Berntsen GK, Schuster T, Henriksen E, Horsch A. Electronic symptom reporting between patient and provider for improved health care service quality: a systematic review of randomized controlled trials. Part 2: methodological quality and effects. J Med Internet Res. 2012;14(5):e126.

5. Thong MSY, Kaptein AA, Benyamini Y, Krediet RT, Boeschoten EW, Dekker FW. Association between a self-rated health question and mortality in young and old dialysis patients: a cohort study. Am J Kidney Dis. 2008;52(1):111-7.

6. Finkelstein FO, Wuerth D, Finkelstein $\mathrm{SH}$. Health related quality of life and the CKD patient: challenges for the nephrology community. Kidney Int. 2009:76(9):946-52.

7. Grincenkov FR, Fernandes N, dos Santos Pereira B, Bastos K, Lopes AA, Finkelstein $\mathrm{FO}$, et al. Impact of baseline health-related quality of life scores on survival of incident patients on peritoneal dialysis: a cohort study. Nephron. 2015:129(2):97-103.

8. Bjorner JB, Kreiner S, Ware JE, Damsgaard MT, Bech P. Differential item functioning in the Danish translation of the SF-36. J Clin Epidemiol. 1998;51(11):1189-202.

9. Rabin R, De Charro F. EQ-5D: a measure of health status from the EuroQol Group. Ann Med. 2001;33(5):337-43.

10. Hays RD, Kallich J, Mapes D, Coons S, Amin N, Carter WB, Kamberg C. Kidney Disease Quality of Life Short Form (KDQOL-SF ${ }^{\mathrm{TM}}$ ), Version 1.3: a manual for use and scoring, Santa Monica, Calif.: RAND Corporation. 1997. https://www.rand.org/pubs/papers/P7994.html. Accessed 05 Feb 2018.

11. Schougaard LM, Larsen $L P$, Jessen $A$, Sidenius $P$, Dorflinger $L$, de Thurah A, et al. AmbuFlex: tele-patient-reported outcomes (telePRO) as the basis for follow-up in chronic and malignant diseases. Qual Life Res. 2016;25(3):525-34.

12. Danish Nephrology Registry. Annual report 2015. Copenhagen, 2015. http://www.nephrology.dk/Publikationer/Landsregister/\%C3\%85rsrappor t\%202015.pdf. Accessed 24 Oct 2016.

13. Charlson ME, Pompei P, Ales KA, Mackenzie CR. A new method of classifying prognostic comorbidity in longitudinal studies: development and validation. J Chronic Dis. 1987;40(5):373-83.

14. Levey AS, Greene T, Beck GJ, Caggiula AW, Kusek JW, Hunsicker LG, et al. Dietary protein restriction and the progression of chronic renal disease: what have all of the results of the MDRD study shown? Modification of Diet in Renal Disease Study group. J Am Soc Nephrol. 1999:10(11):2426-39.

15. Gama-Axelsson T, Lindholm B, Bárány $P$, Heimbürger $O$, Stenvinkel $P$, Qureshi AR. Self-rated appetite as a predictor of mortality in patients with stage 5 chronic kidney disease. J Renal Nutr. 2013;23(2):106-13.

16. Kaplan EL, Meier P. Nonparametric estimation from incomplete observations. J Am Stat Assoc. 1958:53(252):457-81.

17. Robinson-Cohen C, Hall YN, Katz R, Rivara MB, de Boer IH, Kestenbaum BR, et al. Self-rated health and adverse events in CKD. Clin J Am Soc Nephrol. 2014;9(12):2044-51

18. Han SS, Kim KW, Na KY, Chae DW, Kim YS, Kim S, et al. Quality of life and mortality from a nephrologist's view: a prospective observational study. BMC Nephrol. 2009:10:39.

19. Porter AC, Lash JP, Xie D, Pan Q, DeLuca J, Kanthety R, et al. Predictors and outcomes of health-related quality of life in adults with CKD. Clin J Am Soc Nephrol. 2016;11(7):1154-62.

20. Burrowes JD, Larive B, Chertow GM, Cockram DB, Dwyer JT, Greene T, et al. Self-reported appetite, hospitalization and death in haemodialysis patients: findings from the Hemodialysis (HEMO) Study. Nephrol Dial Transplant. 2005:20(12):2765-74.

21. Kwon YE, Kee YK, Yoon CY, Han IM, Han SG, Park KS, et al. Change of nutritional status assessed using subjective global assessment is associated with all-cause mortality in incident dialysis patients. Medicine (Baltimore) 2016:95(7):e2714.

22. Fukuhara S, Yamazaki S, Marumo F, Akiba T, Akizawa T, Fujimi S, et al. Health-related quality of life of predialysis patients with chronic renal failure. Nephron Clin Pract. 2007;105(1):C1-8.

23. Jesky MD, Dutton M, Dasgupta I, Yadav P, Ng KP, Fenton A, et al. Healthrelated quality of life impacts mortality but not progression to end-stage renal disease in pre-dialysis chronic kidney disease: a prospective observational study. PLoS ONE. 2016;11(11):e0165675. 